\title{
Analysis of Structure Transformations in Rail Surface Induced by Plastic Deformation via Barkhausen Noise Emission
}

\author{
M. Neslušan ${ }^{a, *}$, K. ZGÚtová ${ }^{b}$, K. KolaŘÍK ${ }^{c}$, J. ŠRAmeK $^{b}$, J. ČAPeK ${ }^{c}$ \\ ${ }^{a}$ Faculty of Mechanical Engineering, University of Žilina, Univerzitná 1, 01026 Žilina, Slovakia \\ ${ }^{b}$ Faculty of Civil Engineering, University of Žilina, Univerzitná 1, 01026 Žilina, Slovakia \\ ${ }^{c}$ Faculty of Nuclear Science and Physical Engineering, ČVUT Praha, Trojanova 13, 12000 Praha, Czech Republic

\begin{abstract}
This paper reports about magnetic Barkhausen noise emission of the rail surface exposed to long time cyclic plastic deformation. Severe plastic deformation of the rail surface induces remarkable structure transformations and alterations of stress state which contribute to valuable decrease of the Barkhausen noise emission compared to untouched surface of the rail. The paper analyses correlation between the Barkhausen noise signals (as well as extracted Barkhausen noise envelopes) and surface state (expressed in terms of micrographs, microhardness readings and residual stresses). This study would contribute to a possible concept for preventing unexpected rails
\end{abstract} \\ deformation (or cracking) due to their thermal dilatation via the Barkhausen noise technique.
}

DOI: 10.12693/APhysPolA.131.1099

PACS/topics: 75.60.Ej

\section{Introduction}

Large axle loads of the trains usually cause serious wear and structure transformations of the rails. White etching layer (WEL) is a typical defect in the surface region of the rail originating from repetitive and severe plastic deformation [1]. $W E L$ is a generic term for very hard surface layer which appears white on micrographs due to high resistance against etching. It was reported that $W E L$ in the surface of rails is inhomogeneous and composed of several different microstructures $[2,3]$. WEL as a hard structure may lead to the brittle cracking and subsequent fatigue crack propagation. It was reported [1] that $W E L$ is a product of rolling-contact fatigue caused by the alternating stresses associated with rolling contact bodies. Operation of the rails containing $W E L$ regions might be risky with the respect to possible rails macrocracking initiated by microcracks in the WEL. Furthermore, rails are subjected to grinding process in order to remove damaged layer and surface asperities [4]. For these reasons, the suitable non destructive method would be beneficial for assessment of a degree of the rail surface damage. Magnetic Barkhausen noise $(M B N)$ is sensitive to microstructure and stress state. Being so, this study deals with sensitivity of $M B N$ for such purpose (as the promising technique for monitoring rails surface [5]).

\section{Experimental}

The experimental study was carried out on hot rolled rail steel R220 (strength $785 \pm 50 \mathrm{MPa}$, hardness $215 \pm$ $35 \mathrm{HB}$ ) of chemical composition indicated in Table I. The rail was subjected to the 20 years accumulated passing tonnage about 0.8 million tons per year. Figure 1 shows

\footnotetext{
* corresponding author; e-mail:

miroslav.neslusan@fstroj.uniza.sk
}

a photo of the rail profile with indication of the analyzed surface.

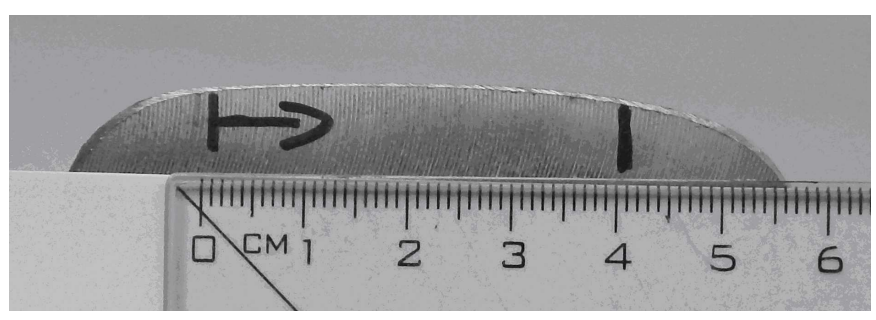

Fig. 1. The rail cross-section with indication of measured width.

TABLE I

Chemical composition of the rail steel R220 in wt\%.

\begin{tabular}{c|c|c|c|c|c|c|c}
\hline \hline $\mathrm{Fe}$ & $\mathrm{C}$ & $\mathrm{Mn}$ & $\mathrm{Si}$ & $\mathrm{Cr}$ & $\mathrm{Ni}$ & $\mathrm{Cu}$ & $\mathrm{P}$ \\
\hline bal. & 0.60 & 1.05 & 0.21 & 0.12 & 0.13 & 0.07 & 0.02
\end{tabular}

MBN was measured using RollScan 350 and software $\mu$ Scan 500 (mag. voltage $5 \mathrm{~V}$, mag. frequency $125 \mathrm{~Hz}$, 10 bursts, sensor type S1-18-12-01, frequency range of $M B N$ from 10 to $1000 \mathrm{kHz}) . M B N$ values were obtained by averaging $10 M B N$ bursts (5 magnetizing cycles). $M B N$ refers to the rms (effective) value of the signal. Residual stresses and phase analysis were measured via X-ray diffraction technique $(\mathrm{XRD})(\{211\}, \alpha$-Fe, Cr $K_{\alpha}, 40 \mathrm{kV}, 30 \mathrm{~mA}$, average sensing depth approximately $5 \mu \mathrm{m}$, X'Pert PRO). $10 \mathrm{~mm}$ long pieces were routinely prepared for metallographic observations (etched by $3 \%$ Nital for $8 \mathrm{~s}$ ) to reveal the microstructure transformations induced by severe plastic deformation. Microstructure was observed in the direction perpendicular against the track direction.

The Vickers microhardness readings were conducted by Zwick Roel ZHm micro-hardness tester by applying the $50 \mathrm{~g}$ load for $10 \mathrm{~s}$. Microhardness was determined 
by averaging 3 repetitive measurements (3 microhardness profiles spaced $0.1 \mathrm{~mm}$ ). All measurements were conducted at 7 points within the rail surface indicated in Fig. 1. The first position was placed $3 \mathrm{~mm}$ from the left side. The next positions were spaced $6 \mathrm{~mm}$ each to other. $5 \mathrm{~mm}$ surface layer was removed by milling to investigate bulk MBN. Afterwards $0.2 \mathrm{~mm}$ layer was electrolytically etched to remove surface alterations initiated by previous milling.

\section{Results and discussion}

Figure 2 shows micrographs of the rail surface containing WEL. This layer is resistant against etching and can be easily contrasted against the deeper pearlite regions appearing dark. Figure 2 also shows that $W E L$ can occur on the rail surface as the localized spots (Fig. 2 top, the same character can be found at the positions 3,21 , and $27 \mathrm{~mm}$ ) or the continuous layer of variable thickness (Fig. 2 bottom - the positions 9 and $15 \mathrm{~mm}$ ). Bulk, the positions 33 and $39 \mathrm{~mm}$ are free of WEL. It was found that $W E L$ has the martensite structure formed after austenitization due to temperature rise caused by frictional heating in the rail-wheel contact areas [1].

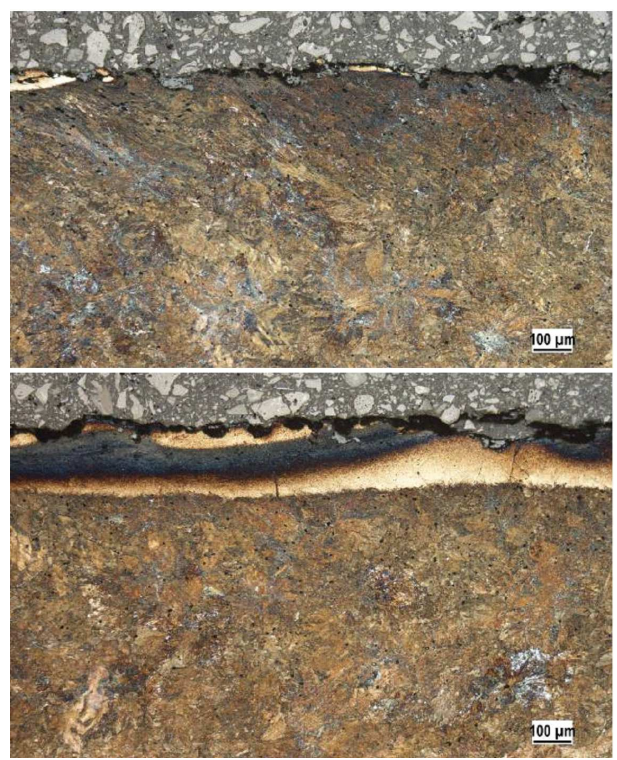

Fig. 2. Micrographs of the rail, Nital 3\%: (top) micrograph of the rail surface $-3 \mathrm{~mm}$ from left side, (bottom) micrograph of the rail surface $-15 \mathrm{~mm}$ from left side.

Information about martensite structure corresponds with the high hardness of $W E L$ (see Fig. 3) as well as the phase analysis carried out using XRD technique. Regions of thick $W E L$ are subjected to the main load of the rail containing retained austenite (as an evidence of heating above the austenitizing temperature) and the high degree of martensite tetragonality. The phase analysis and previous papers $[2,6]$ indicate that cementite is completely decomposed in all surface regions and especially in the thick $W E L$ carbon is embedded in the matrix in the supersaturated state (solid solution of carbon).

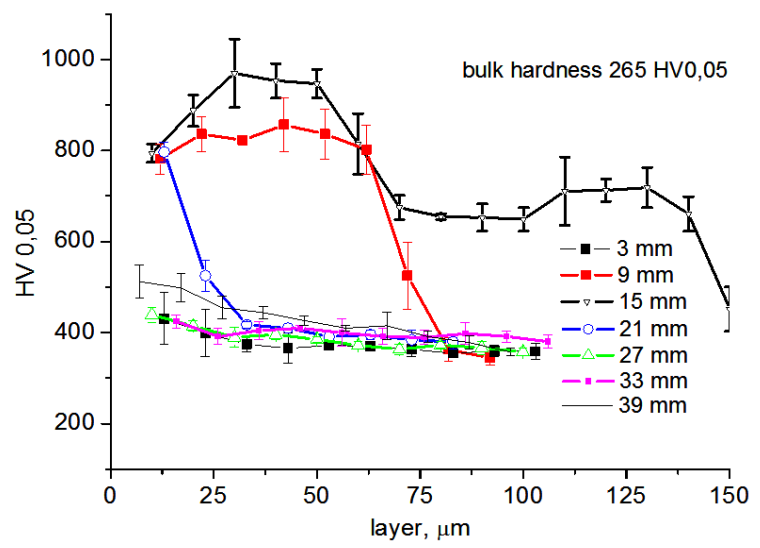

Fig. 3. Microhardness profiles.

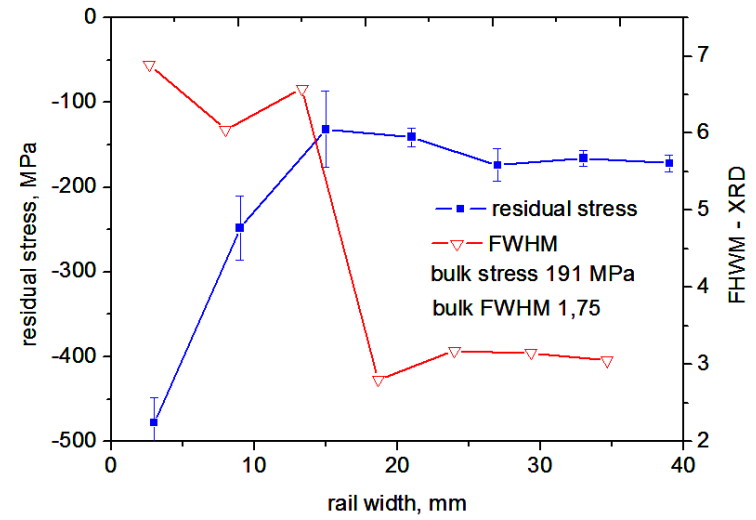

Fig. 4. Residual stresses and FWHM (XRD technique).

Figure 4 shows that the regions containing retained austenite and the high degree of martensite tetragonality exhibit broadened diffraction XRD peaks expressed in term of full width at half maximum (FWHM) and higher magnitude of compressive stresses which correspond with the high $W E L$ hardness. However, the magnitude of residual stresses tends to decrease along with the increasing thickness of $W E L$ due to dynamic recovery and tempering effect of the repeated cyclic loading on the previously initiated WEL. Compared to the XRD technique, the $M B N$ technique exhibits better sensitivity against the $W E L$ thickness (see Fig. 5). Thick WEL corresponds with the low $M B N$ and vice versa. Relation between $M B N$ and $W E L$ thickness is linear and can be expressed as follows:

$$
M B N=-1.9 W E L_{\text {thickness }}+433 .
$$

The Pearson coefficient of correlation is -0.99 . $M B N$ as a function of stresses is expressed in Eq. (2):

$$
M B N=-0.55 \sigma-71 \text {. }
$$

The Pearson coefficient of correlation is only -0.41 . The main reason can be viewed in the different sensing depths of XRD and $M B N$ techniques. XRD penetration depth is only about $5 \mu \mathrm{m}$ whereas $M B N$ is more sensitive to the deeper extent of surface damage (about $40 \mu \mathrm{m}$ ). Remarkably lower $M B N$ in the thick $W E L$ are due to 
1. martensite matrix of the high degree of lattice tetragonality containing carbon in the supersaturated state and the corresponding high hardness,

2. retained austenite in the $W E L$ (see Table II),

3. oxides ( $\mathrm{FeO}$ and $\mathrm{Fe}_{2} \mathrm{O}_{3}$ ) embedded in the martensite matrix as hard ferromagnetic particles strongly pinning BWs motion (see Table II),

4. microcracks as the defects not producing $M B N$ emission,

5. harder propagation of $M B N$ signal through the hard-magnetic WEL.

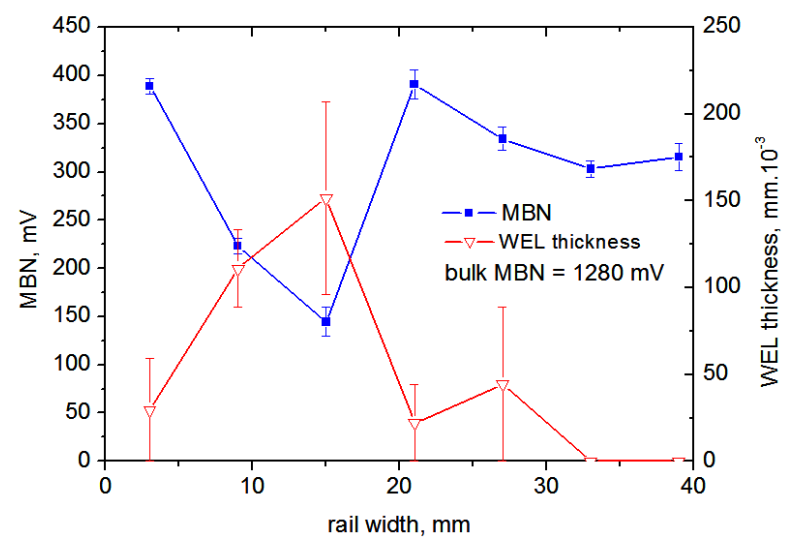

Fig. 5. $M B N$ and $W E L$ thickness through the rail width.

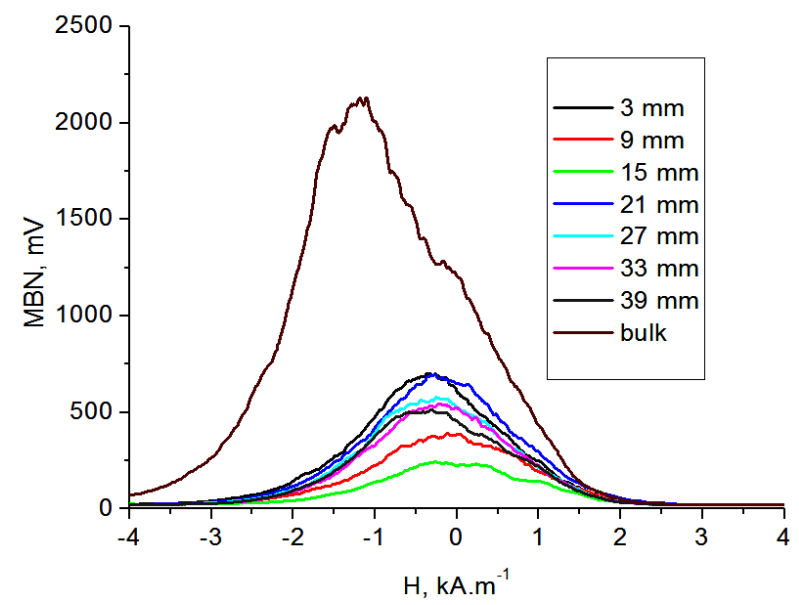

Fig. 6. $M B N$ envelopes.

The high pinning strength of the thick $W E L$ regions can be also indicated by the use of $M B N$ envelopes as those illustrated in Fig. 6. $M B N$ envelopes for thick $W E L$ are shifted to the higher magnetic fields and their envelopes are broadened (also peak height is decreased).

On the other hand, bulk structure emits significantly higher $M B N$ compared either with the $W E L$ or the whole rail surface. This behavior can be linked with the pearlite matrix of lower dislocation density (corresponding with the lower hardness) due to the low degree of plastic deformation (the rail surface undergoes severe plastic deformation during hot rolling), the absence of oxides and retained austenite in the structure. The bulk $M B N$ envelope exhibits remarkably higher maximum shifted to the lower magnetic field as opposed to the whole rail surface.

TABLE II

Phases on the rail surface indicated by XRD technique

\begin{tabular}{|c|c|c|c|c|c|c|c|}
\hline \multirow{2}{*}{$\begin{array}{l}\text { Dis. } \\
{[\mathrm{mm}]}\end{array}$} & \multicolumn{2}{|c|}{$\mathrm{Fe}$} & \multirow[t]{2}{*}{$\mathrm{Fe}_{3} \mathrm{C}$} & \multirow[t]{2}{*}{$\mathrm{Fe}_{2} \mathrm{O}_{3}$} & \multirow[t]{2}{*}{$\mathrm{FeO}$} & \multirow[t]{2}{*}{$\mathrm{C}$} & \multirow{2}{*}{$\begin{array}{l}\mathrm{Fe} \\
\mathrm{fct}\end{array}$} \\
\hline & $\alpha$ & $\gamma$ & & & & & \\
\hline 3 & - & $\bullet$ & - & • & $\bullet$ & $\bullet$ & $\bullet^{+}$ \\
\hline 9 & - & $\bullet$ & - & - & $\bullet$ & $\bullet$ & $\bullet^{+}$ \\
\hline 15 & - & $\bullet$ & - & $\bullet$ & $\bullet$ & $\bullet$ & $\bullet^{+}$ \\
\hline 21 & - & - & - & $\bullet$ & - & $\bullet$ & $\bullet^{+}$ \\
\hline 27 & - & - & - & - & - & • & $\bullet^{-}$ \\
\hline 33 & - & - & - & - & - & $\bullet$ & $\bullet^{-}$ \\
\hline 39 & - & - & - & - & - & $\bullet$ & $\bullet^{-}$ \\
\hline bulk & $\bullet$ & - & $\bullet$ & - & - & - & - \\
\hline
\end{tabular}

\section{Conclusions}

Microcracking in the hard and brittle $W E L$ arises from the cyclic long term loading and potentially can initiate rail macrocracking. For this reason, rails should be subjected to the re-grinding process in order to remove the damaged layer and refresh the rail surface. $M B N$ technique could be potentially employed for assessment of $W E L$ thickness as the layer removed by grinding (see Eq. (1)). Finally, this paper is the pilot study which suggest the possible concept to prevent the unexpected rails deformation (or cracking) due to their thermal dilatation (and the corresponding stresses) initiated by the ambient temperature fluctuations via $M B N$ technique. However, $M B N$ emission initiated by external stresses superimposes with $M B N$ from the accumulated damage of the rail surface due to its cyclic severe loading.

\section{Acknowledgments}

This work was supported by the VEGA project no. $1 / 0254 / 15$

\section{References}

[1] S. Pal, J.T. Daniel, H.G. Valente, A. Wilson, A. Atrens, Eng. Fail. Anal. 22, 152 (2012).

[2] J. Takahashi, K. Kawakami, M. Ueda, Acta Mater. 58, 3602 (2010).

[3] W. Österle, H. Rooch, A. Pyzalla, L. Wang, Mater. Sci. Eng. A 303, 150 (2001).

[4] J.F. Santa, A. Toro, R. Lewis, Tribo. Int. 95, 5 (2016).

[5] RailScan, Metalelektro Méréstechnika, Budapest 2017.

[6] L. Wang, A. Pyzalla, W. Stadlbauer, E.A. Werner, Mater. Eng. A 359, 31 (2003). 